\title{
Burnout and its Organizational Effects: A Study on Literature Review
}

\section{Rahat Ahmed Chowdhury*}

Department of Business Administration, Leading University, Bangladesh

\begin{abstract}
Job Burnout or Employee burnout is one of the most significant emerging issues dealing by organizations. In the manufacturing and service industry employees that are constantly subjected to a challenging work environment may experience increased levels of job stress and burnout or even leave the industry entirely. This research aims to study the effect of Burnout on Organization. This study conducted for research demand and it strived to fill the gap by compiling ten research studies conducted so far on job burnout and occupational stress all over the world. The present study proposed some areas where future studies can be carried out.
\end{abstract}

Keywords: Stress; Job burnout; Job satisfaction; Cynicism; Emotional exhaustion

\section{Introduction}

Burnout is a prolonged response to chronic emotional and interpersonal stressors on the job, and is defined by the three dimensions of exhaustion, cynicism, and inefficacy. The past 25 years of research has established the complexity of the construct and places the individual stress experience within a larger organizational context of people's relation to their work. Recently, the work on burnout has expanded internationally and has led to new conceptual models. The focus on engagement, the positive antithesis of burnout, promises to yield new perspectives on the interventions to alleviate burnout. The social focus of burnout, the solid research basis concerning the syndrome, and its specific ties to the work domain make a distinct and valuable contribution to people's health and well-being [1].

\section{Statement of the problem and rationale of the study}

Occupational stress and Job Burnout is an emerging concept. Many researches so far conducted to reveal the actual effects of job burnout on organizational performance. This study strived to fill the gap by presenting a substantial number of research studies conducted so far in different aspects of burnout. This study would benefit the researchers, practitioners, policy makers, students, and various stakeholders. This study would definitely augment the current research on job burnout.

\section{Objective of the Study}

The purpose of this paper is to examine the relationship between job burnout (emotional exhaustion and depersonalization) and its organizational effect. The study should enhance further research and this study will be conducted based on several studies conducted on research topic.

\section{Research Methodology}

The methodology used for this study was literature survey. The study was completely based on compiling the studies conducted so far on burnout. The researchers collected different studies on Burnout from different libraries, universities, and book stores. In reviewing the literature, both theoretical and empirical studies were taken into consideration. In total, 10 studies were collected for the review.

\section{Burnout}

The term "burnout" was coined to describe a psychological syndrome that is characterised by a negative emotional reaction to one's job as a consequence of extended exposure to a stressful work environment
$[1,2]$. The initial research on burnout, which was conducted in the 1970 s using interviews, surveys and field observations, focused primarily on individuals working in the human services professions, such as health care, social services, education and legal services [2-4]. However, over the years, burnout has become a phenomenon of notable global significance and it is recognised that it affects individuals in a wide range of occupations [5]. Maslach and Jackson conceptualise burnout as comprising of three dimensions, namely, emotional exhaustion, depersonalisation and reduced personal accomplishment that can occur among individuals that work with people in some capacity [6].

Types of burnout: Maslach describes three dimensions of burnout:

- Exhaustion: Feeling overextended, both emotionally and physically

- Cynicism: Taking a cold, cynical attitude toward responsibilities.

- Ineffectiveness: When people feel ineffective, they feel a growing sense of inadequacy

- Exhaustion: Exhaustion is the central quality of burnout and the most obvious manifestation of this complex syndrome. When people describe themselves or others as experiencing burnout, they are most often referring to the experience of exhaustion.

Of the three aspects of burnout, exhaustion is the most widely reported and the most thoroughly analyzed. The strong identification of exhaustion with burnout has led some to argue that the other two aspects of the syndrome are incidental or unnecessary [7]. However, the fact that exhaustion is a necessary criterion for burnout does not mean it is sufficient. If one were to look at burnout out of context, and simply focus on the individual exhaustion component, one would lose sight of the phenomenon entirely.

Although exhaustion reflects the stress dimension of burnout, it fails to capture the critical aspects of the relationship people have with their work. Exhaustion is not something that is simply experienced-

*Corresponding author: Rahat Ahmed Chowdhury, Department of Business Administration, Leading University, Sylhet-3100, Bangladesh, Tel: +8801911528681; E-mail: rac.onlinebd@gmail.com

Received September 29, 2018; Accepted October 17, 2018; Published October 27, 2018

Citation: Chowdhury RA (2018) Burnout and its Organizational Effects: A Study on Literature Review. J Bus Fin Aff 7: 353. doi: 10.4172/2167-0234.1000353

Copyright: ( 2018 Chowdhury RA. This is an open-access article distributed under the terms of the Creative Commons Attribution License, which permits unrestricted use, distribution, and reproduction in any medium, provided the original author and source are credited. 
rather, it prompts actions to distance oneself emotionally and cognitively from one's work, presumably as a way to cope with the work overload. Within the human services, the emotional demands of the work can exhaust a service provider's capacity to be involved with, and responsive to, the needs of service recipients.

Depersonalization/cynicism: Depersonalization is an attempt to put distance between oneself and service recipients by actively ignoring the qualities that make them unique and engaging people. Their demands are more manageable when they are considered impersonal objects of one's work. Outside of the human services, people use cognitive distancing by developing an indifference or cynical attitude when they are exhausted and discouraged. Distancing is such an immediate reaction to exhaustion that a strong relationship from exhaustion to cynicism (depersonalization) is found consistently in burnout research, across a wide range of organizational and occupational settings.

Ineffectiveness/inefficacy: The relationship of inefficacy (reduced personal accomplishment) to the other two aspects of burnout is somewhat more complex. In some instances it appears to be a function, to some degree, of either exhaustion, cynicism, or a combination of the two [8]. A work situation with chronic, overwhelming demands that contribute to exhaustion or cynicism is likely to erode one's sense of effectiveness. Further, exhaustion or depersonalization interfere with effectiveness: It is difficult to gain a sense of accomplishment when feeling exhausted or when helping people toward whom one is indifferent. However, in other job contexts, inefficacy appears to develop in parallel with the other two burnout aspects, rather than sequentially. The lack of efficacy seems to arise more clearly from a lack of relevant resources, whereas exhaustion and cynicism emerge from the presence of work overload and social conflict.

Effect of burnout: The consequences of burnout can be of two types: individual related and the effect on organization's overall productivity. Tennant has explained the personal consequences such as depressive disorder arising from stress. The effect on organizational of employee burnout includes decrease in employee's job performance and job satisfaction, diminished organizational commitment and increase in employee's absenteeism and turnover [9]. A review and an integration of research on job burnout.

\section{Burnout and job satisfaction}

Maslach et al. [10], have studied the relationship of job satisfaction with burnout; and have found burnout to be a strongly related to job dissatisfaction. With-in three phases of burnout, emotional exhaustion is found to be more significant cause of job dissatisfaction than cynicism. Burnout and job satisfaction in New Zealand psychiatrists: a national study.

\section{Burnout and organizational commitment}

Meyer and Allen have defined three types of organizational commitment: affective, continuance and normative. Affective commitment is the emotional attachment and identification of employees with their organization. Continuance commitment involves the cost of leaving the organization; while normative commitment is the sense of obligation of the employees to stay in the organization. Several research studies have reported that burnout reduced employee's organizational commitment $[9,10]$. A review and an integration of research on job burnout. Within the three dimensions of burnout, emotional exhaustion and cynicism were found strongly associated with diminished organizational commitment. Burnout in Organizational Life. The Influence of Demographics on Job Burnout.

\section{Burnout and turnover intention}

Leiter and Maslach [5] have reported positive relationship of burnout and turnover intention. Several authors have tested the positive relationship of burnout and intention to turnover $[5,6]$. Schaufeli and Bakker have investigated employee's turnover intention and burnout in multiple settings; insurance companies, pension funds, an occupational health and home care institution. They have confirmed the positive relationship between burnout and turnover intention. Goodman and Boss reported that employees who left the organization scored higher on burnout than those who chose to stay.

\section{Organizational outcomes of burnout}

One of the major problems of burnout is that it creates conflict within the organization often it is termed as work family conflict Work family conflict occurs when the demands of work interfere with the ability to perform family duties [11]. Work family conflict is linked to adverse outcomes, including lower job productivity and satisfaction, poorer mental and physical health, and higher burnout. The conservation of resources (COR) theory has been applied in numerous studies to understand the causes and consequences of Work family conflict $[12,13]$. According to conservation of resources theory, individuals seek to retain, gain, or avoid losing, valued resources such as personal health, stable employment, and support from coworkers [12]. Competing demands from work and family role promote resources loss, which is a major source of stress. Prolonged Work family conflict can lead to poor health outcomes such as burnout and depression [14]. Work can also benefit individuals and their families [11]. Work family efficiency is a process that occurs when work-related experiences generate or promote the development of resources (e.g. mood, psychosocial benefits) that benefit the family domain [15]. Research shows that higher Work family efficiency is associated with positive outcomes, including higher job satisfaction, and improved physical health and mental health [16]. The resource gain-development (RGD) model provides a framework for understanding Work family efficiency [15]. The resource gain-development model assumes that individuals have a natural predisposition to developing, achieving, and growing to the greatest degree possible for themselves and groups or systems they belong to, including family and organizations [15]. According to the resource gain-development model, Work family efficiency occurs when resources gained in the work domain are applied, sustained, and reinforced in the family domain. The extent of enrichment experienced is dependent on the level of resources an individual already possesses. For example, compared to mothers with few resources, mothers with high resource levels (e.g. high income or a supportive partner) can more readily acquire additional resources, and consequently experience greater Work family efficiency. Building on past studies showing that work-family profiles have differing implications for indicators of health and well-being [17], the final aim of this paper was to examine the relationships between work-to family profiles and burnout. Previous research shows that compared to the active and contradictory profiles, the beneficial profile had the highest life satisfaction and the lowest psychological strains. Job and life satisfaction, core self-evaluation, and job exhaustion have also differed across work-family profiles [17]. Distinct profiles of Work family conflict and Work family efficiency may have implications for burnout, which represents a "combination of physical fatigue, emotional exhaustion, and cognitive weariness" [7]. Existing studies have demonstrated that Work family conflict is associated with burnout [18], which affects work performance and parenting, and is a growing problem, particularly for women employees. The associations 
between Work family conflict and burnout can be understood within the context of conservation of resources theory. Work family conflict reflects a process whereby work-related demands lead to a threatened, or actual loss, of personal resources, leading to stress [13]. Resource losses are then exacerbated as individuals in vest a variable resources to prevent further losses, leading to a spiral of resource losses, and over time burnout [12]. It is then plausible that profiles characterized by higher levels of Work family conflict will experience higher burnout levels than profiles with lower Work family conflict levels. In contrast, Work family efficiency has been linked with lower levels of burnout [18]. According to conservation of resources theory, in times of lowstress individuals seek to gain surplus resources in order to prevent or minimize future losses [12]. Moreover, any gains can at least partially offset stress and potentially minimize burnout; thus, suggesting that Work family efficiency may serve as a buffer against the adverse effects of Work family conflict. It is then plausible that individuals with high Work family efficiency may not experience the effects of Work family conflict to the same extent as those with low Work family efficiency.

\section{Findings \& Recommendation}

\section{Findings}

Job burnout is highly related with the factor emotional exhaustion. The relation between work family conflict, intention to leave organization and job burnout is also positive. If job burnout level is decreases, then the work family conflict and intention to leave organization level will be increases.

\section{Recommendation}

The importance of identifying the most important factors in workers' burnout, and of designing an effective questionnaire to ascertain the level and type of burnout in individual workers, is crucial if management is to be able to implement appropriate strategies of prevention and/or alleviation of stressful situations, or provide useful help to valuable workers, once burnout has occurred. Through correct application of such testing, managers can reduce job turnover and the disruption that it causes. Job burnout is highly related to factor emotional exhaustion, organization may take some policy to motivated employees for reduce job burnout. If employee able to reduce their job burnout then job satisfaction and job involvement may also increases.

\section{Conclusion}

Employees' burnout has already been identified as influencing productivity, motivation, intention to leave a job, work family conflict etc. However, burnout is a complex phenomenon and difficult to measure. In this article, three burnout dimensions measures were suggested and utilized to assess their prediction power of a worker's intention to leave a job. High work family conflict was associated with high personal and work burnout, and high work family efficiency was associated low-personal burnout and work burnout. Finally, the inverse relationships between work family efficiency and personal burnout. The potential stressors identified by the study are workload, long working hours, technological problems at work, inadequate salary, and lack of ample time for family and job worries at home. The study also revealed that these stresses are leading to physical and psychological burnout of employees.

\section{References}

1. Maslach C, Schaufeli WB, Leiter MP (2001) Job burnout. Annu Rev Psychol 52: $397-422$

2. Maslach C, Jackson S (1984) Patterns of burnout among a national sample of public contact workers. J Health Hum Resour Adm 7: 189-212.

3. Maslach C (1976) Burned-out. J Hum Behav 5: 16-22.

4. Maslach C, Jackson S (1982) Burnout in health professions: A social psychological analysis. In Sanders G and Suls J. (Eds), Social Psychology of Health and Illness, Erlbaum, Hillsdale, NJ, pp: 227-251.

5. Schaufeli W, Leiter M, Maslach C (2009) Burnout: 35 years of research and practice. Career Development International 14: 204-220.

6. Maslach C, Jackson S (1986) Maslach Burnout Inventory Manual. Consulting Psychologists Press, Palo Alto, CA

7. Ahola K, Vaananen A, Koskinen A, Kouvonen A, Shirom A (2010) Burnout as a predictor of all-cause mortality among industrial employees: a 10-year prospective register-linkage study. J Psychosom Res pp: 1-7.

8. Lee $Y$, Shin $S$ (2010) Job stress evaluation using response surface data mining Int J Ind Ergon 40: 379-385.

9. Cordes C, Dougherty T (1993) A Review and an Integration of Research on Job Burnout. Acad Manag Rev 18: 621-656.

10. Maslach C, Schaufeli W, Leiter MP (2001) Job burnout. Annual Revise Psychology 52: 397-422.

11. Green DE, Walkey FH, Taylor AJW (1985) The three factor structure of the Maslach Burnout Inventory. J Soc Behav Pers 6: 453-472.

12. Hobfoll SE (2001) The influence of culture, community, and the nested-self in the stress process: Advancing conservation of resources theory. Appl Psychol Int Rev 50: 337-421.

13. Grandey AA (1999) Emotion Regulation in the Workplace: A New Way to Conceptualize Emotional Labor. J Occup Health Psychol 5: 95-110.

14. Hobfoll SE, Freedy JR (2001) Conservation of Resources: A general stress theory applied to burnout. J Professional Burnout, pp: 115-129.

15. Wayne JH, Grzywacz JG, Carlson DS, Kacmar KM (2007) Work-family facilitation: a theoretical explanation and model of primary antecedents and consequences. Hum Resour Manag Rev 17: 63-76.

16. McNall LA, Nicklin JM, Masuda AD (2010) A meta-analytic review of the consequences associated with work-family enrichment. J Bus Psychol 25: 381 396.

17. Demerouti E, Bakker A, Nachreiner F, Schaufeli W (2007) The Job Demands Resources Model of Burnout. J Appl Psychol 86: 499-512.

18. Innstrand ST, Espnes GA, Mykletun R (2008) Job Stress, Burnout and Job Satisfaction: An Intervention Study for Staff Working with People with Intellectual Disabilities. J Appl Res Intellect Disabil 17: 119-126. 